\title{
Rapid and High-Fidelity Subsurface Exploration with Multiple Aerial Robots
}

Kshitij Goel, Wennie Tabib, and Nathan Michael

\begin{abstract}
This paper develops a communication-efficient distributed mapping approach for rapid exploration of a cave by a multi-robot team. Subsurface planetary exploration is an unsolved problem challenged by communication, power, and compute constraints. Prior works have addressed the problems of rapid exploration and leveraging multiple systems to increase exploration rate; however, communication considerations have been left largely unaddressed. This paper bridges this gap in the state of the art by developing distributed perceptual modeling that enables high-fidelity mapping while remaining amenable to low-bandwidth communication channels. The approach yields significant gains in exploration rate for multi-robot teams as compared to state-of-the-art approaches. The work is evaluated through simulation studies and hardware experiments in a wild cave in West Virginia.
\end{abstract}

\section{Introduction}

Planetary exploration has benefited from advancements in robotics through automation of data collection for planetary science and robotic precursor missions for human space exploration [1]. To date, robotic precursor missions have engaged in surface exploration of Mars [2] but have not explored subsurface environments despite the potential geological and astrobiological significance of these domains [3, 4]. As a result, robotic subsurface exploration has been identified as a key technology for future missions to these planets [5]. Autonomous navigation and high-resolution perceptual modeling are critical needs in the context of subsurface planetary exploration [6]. A challenge of operating in subsurface environments is communicating to a surface station. Communication may be limited or impossible due to the inability of radio waves to penetrate rock, impeding data relay to Earth, so compact data transmission is critical. Operating on planets far from Earth introduces additional re-

The authors are with the Robotics Institute, Carnegie Mellon University, Pittsburgh, PA 15213 USA (e-mail: \{kgoel1,wtabib,nmichael\}@andrew.cmu.edu) 


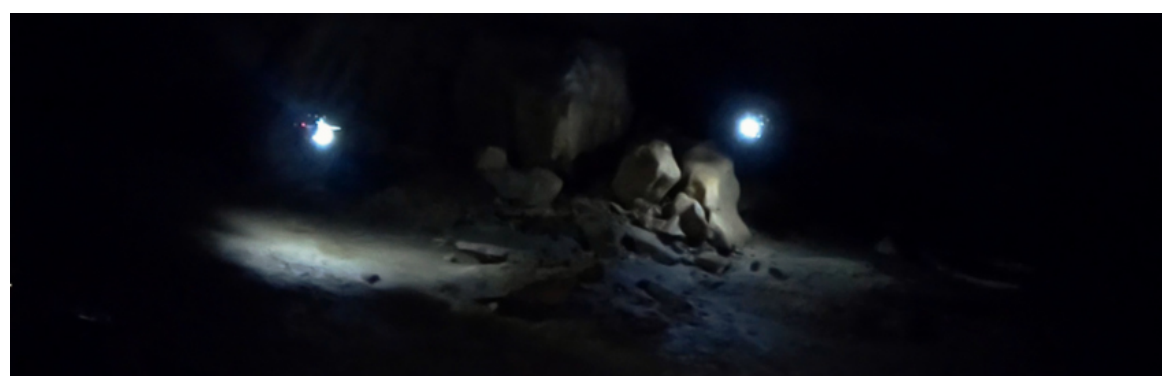

Fig. 1: Cave exploration with two aerial robots in West Virginia, USA. A video of the flight can be accessed at the following link: https://youtu. be/osko8EKKZUM.

strictions on power and compute that may be mitigated by leveraging multiple robots to increase coverage in spatially expansive environments [7]. This work addresses a key challenge for planetary exploration: enabling rapid multi-robot exploration in subsurface environments by leveraging a perceptual modeling framework amenable to low-bandwidth communication while remaining high-fidelity.

Exploration frameworks cannot assume a priori knowledge about the structure of the environment so the exploration system must operate with unknown locomotion constraints. Aerial robots have recently been leveraged to mitigate these constraints in the subterranean domain [8] and considered for subsurface mapping on Mars [3]. In this work, we consider aerial robots operating in a cave on Earth (Fig. 1) as an analog scenario for subsurface exploration on Mars. These robots are often limited by size, weight, and power (SWaP) constraints [8]. Energy constraints on these platforms impose limits on flight endurance necessitating rapid exploration, since the existence of a replenishment infrastructure in the planetary exploration context cannot be guaranteed at these sites [6]. Several frameworks for rapid exploration have been proposed that either use a single fast-moving aerial robot $[9,10,11,12]$ or multiple slow-moving aerial robots $[13,14]$; however, a real-world deployable framework that combines the elements from both is desirable. Such a deployment can potentially be realized by sending multiple aerial robots ("daughtercraft") from a lander ("mothership") to perform rapid, effective, and affordable high-resolution mapping of the target environment, similar to the concept surface mission studied by Matthies [15] for Titan. To this end, a distributed perceptual modeling framework that provides communication-efficient map sharing can enable the daughtercraft team to improve the rate of exploration while transmitting scientific data to the mothership and Earth. The experimental evaluation in this work (Section 3) is motivated by this concept of operations.

Related Work: With the ongoing DARPA Subterranean Challenge [16], there is an increased interest in deploying a team of robots in cave networks. Although existing systems have not shown autonomous operations in a cave, multi-robot exploration systems have been proposed for mine and tunnel environments. These environments differ from cave environments because in most cases the terrain is flat. Notably, teams competing in the challenge have identified that accounting for limited availability of 


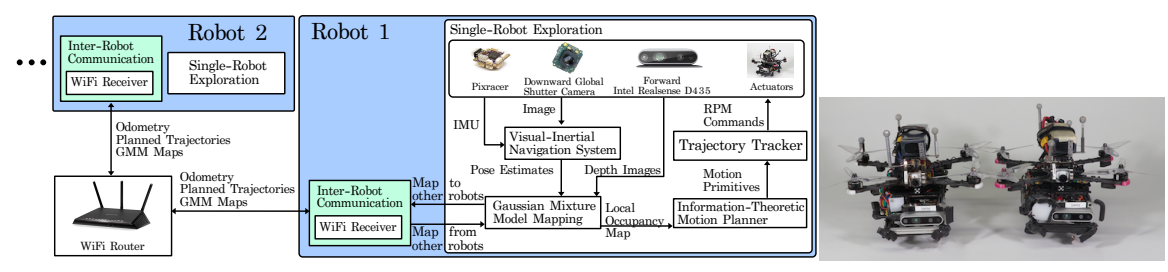

Fig. 2: (Left) Overview of the rapid multi-robot exploration framework and (Right) aerial systems used in experiments in this work.

communication resources within the exploration framework is a key milestone for future work [17, 18, 19]. Ebadi et al. [17] state that the communication bottlenecks faced during mapping with a multi-robot system due to the use of downsampled point clouds can be addressed by map compression techniques or compact representations for motion planning. Dang et al. [18] use the state-of-the-art, memory-efficient OctoMap [20] approach for map representation but mention efficient map sharing as one of the future challenges. Rouček et al. [19] use elevation maps for mapping but only on wheeled and ground robots because the transmission of these maps requires a physically large communication module. Furthermore, the speeds of robots are constrained for these systems. These shared challenges indicate a gap in the state-ofart for communication-efficient distributed mapping methods in rapid aerial multirobot exploration systems for subterranean domains. Corah et al. [14] highlight the benefits of a distributed mapping strategy that exploits the compactness of Gaussian Mixture Models (GMMs) relative to the occupancy grid approach [21]. However, the approach is computationally prohibitive for real-world deployment, limits robot speeds, and the effects of communication constraints on the exploration performance of the robot team are not discussed.

Contributions: We build upon prior single-robot works $[8,10]$ to develop multirobot exploration with the following contributions: (1) a GMM-based distributed mapping approach and occupancy reconstruction for information-theoretic motion planning; (2) an evaluation of the fidelity and memory consumption of the approach against OctoMap [20] and occupancy grid mapping [21]; and (3) a simulation study on the effects of constrained communication on the exploration rate of a two-robot team. All contributions are presented in the context of caves.

\section{Technical Approach}

An overview of the system is shown in Fig. 2. Each robot is equipped with singlerobot exploration and inter-robot communication modules. The exploration module consists of four major subsystems: GMM mapping, information-theoretic motion planning, visual-inertial state estimation, and trajectory tracking. The inter-robot communication module enables sharing information between robots or other com- 


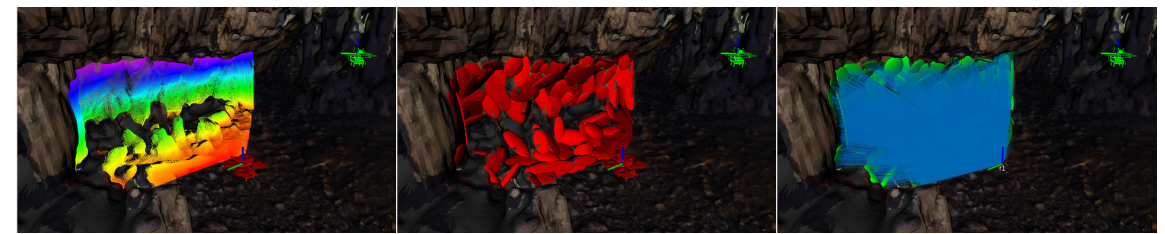

(a)

(b)

(c)

Fig. 3: Overview of the distributed mapping approach. (a) Robot $i$ shown in red, takes a sensor observation shown in colors varying from red to purple and (b) learns a GMM (shown in red). If the GMM is determined to be a keyframe both the GMM and sensor pose are transmitted to robot $j$ (shown in green). (c) The GMM and the sensor pose are transformed into the frame of robot $j$ and used to update the occupancy.

puters on the network. The GMM mapping and planning subsystems together with the communication module constitute distributed mapping (Section 2.1) and multirobot planning (Section 2.2), respectively. In this section, the following mathematical notation is used: lower-case letters represent scalar values, lower-case bold letters represent vectors, upper-case bold letters represent matrices, and script letters represent sets.

\subsection{GMM-based Distributed Mapping}

This section details the distributed mapping approach to share environment models between robots. Consider a team of $N$ robots. At timestep $t$ robot $i \in N$ receives the depth sensor observation, $\mathcal{Z}_{t}^{i}$, which represents a set of points. A Gaussian mixture model (GMM) is learned from these points following the approach from [8]. The GMM is parameterized by $\Theta=\left\{\pi_{m}, \boldsymbol{\mu}_{m}, \boldsymbol{\Sigma}_{m}\right\}_{m=1}^{M}$ where $\boldsymbol{\mu}_{m} \in \mathbb{R}^{3}$ is a mean, $\Sigma_{m} \in \mathbb{R}^{3 \times 3}$ is a covariance, and $\pi_{m} \in \mathbb{R}$ is a weight such that $\sum_{m=1}^{M} \pi_{m}=1$. A GMM representing point set $\mathcal{Z}_{t}^{i}$ is denoted as $\Theta_{\mathcal{Z}_{t}^{i}}$.

Keyframe GMMs: To reduce redundant observations, keyframe GMMs are identified for transmission to other robots. A keyframe GMM, $\hat{\Theta}_{Z_{t}^{i}}$, is determined by approximating the field of view for the current sensor observation as a rectangular pyramid and calculating the overlapping volume with other keyframe fields of view. If the volume is smaller than a user-defined threshold, $\lambda$, the sensor observation is considered to be a keyframe. $\hat{\Theta}_{Z_{t}^{i}}$ and the sensor pose, $\mathbf{S}_{t}^{i} \in \mathrm{SE}(3)$, are transmitted to the other robots or computers on the network.

Each robot maintains its own environment representation and relative initial transforms between robots are assumed to be known. When robot $j$ receives $\hat{\Theta}_{\mathcal{Z}_{t}^{i}}$, it is received in the frame of robot $i$. To transform it into the frame of robot $j$, the relative initial rotation $\mathbf{R}_{0}^{j i} \in \mathbb{R}^{3 \times 3}$ and translation $\mathbf{x}_{0}^{j i} \in \mathbb{R}^{3}$ parameters are applied to the means and covariances of the distribution using the following equations. 


$$
\boldsymbol{\mu}^{j}=\mathbf{R}_{0}^{j i} \boldsymbol{\mu}^{i}+\mathbf{x}_{0}^{j i} \quad \boldsymbol{\Sigma}^{j}=\mathbf{R}_{0}^{j i} \boldsymbol{\Sigma}^{i}\left(\mathbf{R}_{0}^{j i}\right)^{T},
$$

The transformed GMM is incorporated into robot $j$ 's existing GMM map following the approach from $[8,22]$.

Occupancy Reconstruction: A local occupancy grid map $\mathbf{m}_{t}^{i}$ is maintained and centered around the robot's current position $\mathbf{x}_{t}^{i}$ for use in information-theoretic motion planning. To generate $\mathbf{m}_{t}^{i}$, a number of points $\mathbf{p} \in \mathbb{R}^{3}$ equal to the support size, or number of points used to learn the distribution, is sampled and raytraced to the sensor pose $\mathbf{x}_{t}^{i}$. The probability of occupancy along the ray is updated.

Multi-robot Map Updates: Care must be taken to update $\mathbf{m}_{t}^{j}$ when receiving $\hat{\Theta}_{Z_{t}^{i}}$. In addition to applying the transformation parameters so that $\hat{\Theta}_{\mathcal{Z}_{t}^{i}}$ is transformed into the frame of robot $j, \mathbf{m}_{t}^{j}$ must also be updated by sampling points from the transformed $\hat{\Theta}_{Z_{t}^{i}}$ and raytracing through $\mathbf{m}_{t}^{j}$ to the sensor pose, $\mathbf{S}_{t}^{i}$, which must also be transformed into the frame of robot $j$. This ensures the occupancy is updated with observations from both robots. A visualization of this is shown in Fig. 3. Robot $i$ takes a sensor observation (Fig. 3a) and learns $\hat{\Theta}_{\mathcal{Z}_{t}^{i}}$ (Fig. 3b). This keyframe GMM is transmitted to robot $j$, transformed into the frame of robot $j$, and then used to update $\mathbf{m}_{t}^{j}$ (Fig. 3c).

\subsection{Planning for Rapid Multi-Robot Exploration}

Robot $i$ uses $\mathbf{m}_{t}^{i}$ for information-theoretic receding-horizon planning via the strategy presented in [10], which accounts for perception latencies and kinodynamic constraints of the robot. The approach uses Monte Carlo tree search (MCTS) [23] to evaluate the Cauchy-Schwarz Quadratic Mutual Information (CSQMI) [24] for a set of motion primitives over a user-specified time horizon. An informative primitive sequence is selected that maximizes the CSQMI over the MCTS tree. Safety is ensured by checking for collisions with the environment.

The informative trajectories are shared with other robots and inter-robot collision avoidance is enabled through a standard priority-based collision checker assuming a cylindrical robot model [25]. The priorities are assigned manually before the exploration run and remain constant throughout. To reduce the computational complexity for lower priority robots, three optimizations are applied. First, the collision checking is only active when a pair of robots are within a pre-specified radius. To enable this on each robot without assuming a centralized oracle, the robots share odometry information at a sufficiently high rate $(10 \mathrm{~Hz})$ compared to the planning frequency $(1 \mathrm{~Hz})$. Second, the number of cylinders sampled over the planned trajectory is limited to a pre-specified maximum to cap the number of cylinder-cylinder collision checks. This maximum value and the associated cylinder collision radius are selected conservatively based on the length of the motion primitive assuming the robot starts at hover and achieves a top speed at the endpoint. Third, for each robot the collision checks are performed only with the candidate motion primitive and the associated stopping motion primitive at the first depth of the MCTS tree because each depth of 
the tree is of a sufficiently long duration ( $2 \mathrm{~s})$ as compared to the planning time (1s). The inter-robot collision checker is used in the constrained-bandwidth simulation study (Section 3.3).

\section{Experimental Design and Results}

The experimental evaluation is motivated through a concept of operations for a multi-robot exploration mission in a Martian cave. Two robotic systems explore a Martian cave, transmit their maps to a surface station, which serves as a relay to an orbiter, and the orbiter transmits the data to operators on Earth. Three evaluations are conducted to quantify the system performance through this concept of operations: first, the perceptual fidelity and memory usage of the map is compared to state-of-the-art approaches in a representative cave environment (Section 3.1); second, a hardware experiment is demonstrated with two rapidly exploring aerial systems and the communication requirement for each mapping approach is compared (Section 3.2); and third, a simulation study is conducted to study the effects of the bandwidth constraints on exploration performance (Section 3.3).

To correctly analyze the performance of the simulation study, the bottleneck in data transmission rate is identified and bounds on the rates are determined. In this scenario, data is transferred between the subterranean robot and surface station ${ }^{1}$, surface station to orbiter ${ }^{2}$, and orbiter to Earth ${ }^{3}$. The bottleneck in communication is between the subterranean robot and surface station when the robot is transmitting at depths between 20-25 m below ground, so the results in Section 3.3 are presented for the rates $0.1-0.25 \mathrm{Mbit} / \mathrm{s}$, which are in line with data transmission rates at these depths. Throughout this section the shorthand OG is used to refer to the occupancy grid mapping approach [21] while OM refers to OctoMap [20].

\subsection{Perceptual Detail Evaluation}

The first evaluation compares the perceptual fidelity of different environment representations in the context of memory usage. An RGB image and point cloud of a crevice in the cave are shown in Figs. $4 \mathrm{a}$ and $4 \mathrm{~b}$ respectively. It is not clear from the

\footnotetext{
1 Whittaker et al. [6] suggest the use of either very low frequency (VLF) radios or magnetoinductive (MI) links to achieve limited data rate through thick layers of rock. The MI links in particular can provide approximately 20-25 m dry soil penetration at channel capacity ranging from 0.1-0.25 Mbit/s when using small antennas (coils) [26]. In the results presented in Section 3.3, it is assumed that the robots could be equipped with these MI links.

2 Orbiters can communicate at approximately $0.208-0.521 \mathrm{Mbit} / \mathrm{s}$ with a surface station for 8 minutes per sol, or Martian day [27].

3 To transmit from the orbiter to Earth, the communication rate depends on which orbiter is above the lander to relay the data to Earth. The simulation study in Section 3.3 assumes the lowest data rate from the Mars Odyssey orbiter, which ranges from 0.128-0.256 Mbit/s [27].
} 


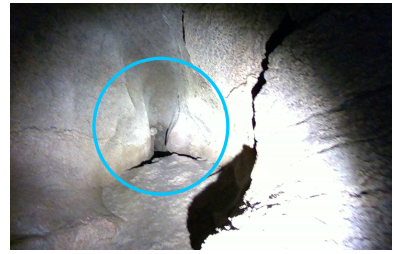

(a) RGB Image

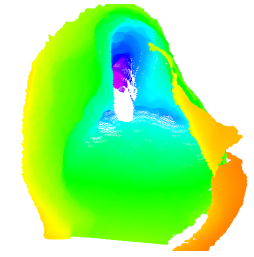

(b) Point Cloud

\begin{tabular}{c|c|c|c}
\hline & $0.025 \mathrm{~m}$ & $0.05 \mathrm{~m}$ & $0.1 \mathrm{~m}$ \\
\hline (bytes) & (bytes) & (bytes) & (bytes) \\
\hline GMM & \multicolumn{3}{|c}{ Occupancy Grid (OG) } \\
4028 & $1.3 \times 10^{6} \mid 1.8 \times 10^{5}$ & $2.7 \times 10^{4}$ \\
\hline GMM & \multicolumn{3}{|c}{ OctoMap (OM) } \\
4028 & $2.2 \times 10^{5}\left|5.8 \times 10^{4}\right| 1.4 \times 10^{4}$ \\
\hline
\end{tabular}

(c) Memory

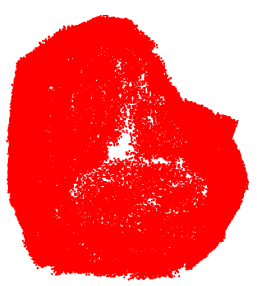

(d) Resampled GMM

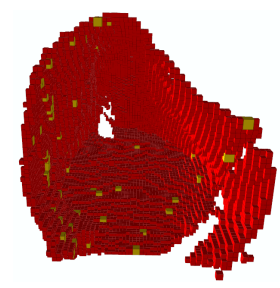

(e) $\mathrm{OM}(0.025 \mathrm{~m})$

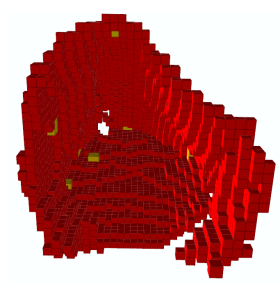

(f) $\mathrm{OM}(0.05 \mathrm{~m})$

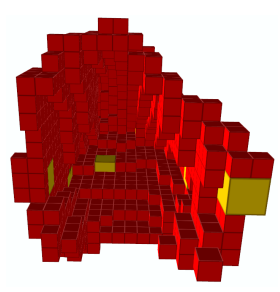

(g) $\mathrm{OM}(0.1 \mathrm{~m})$

Fig. 4: Fidelity and memory usage evaluation of several mapping approaches. (a) and (b) illustrate data from a representative environment the robot may encounter in the cave. A potential passage is circled in cyan. (c) highlights significant reduction in memory usage required by the GMM approach as compared to the OG and OM approaches. (d) Resampled points from the GMM are shown in red. (e)-(g) illustrate the OctoMap representation with leaf sizes varying from $0.025 \mathrm{~m}$ to $0.1 \mathrm{~m}$. Leaf voxels are shown in red and larger voxels in yellow.

image and depth information if the passage continues or there is a lack of data due to insufficient accuracy in the sensor observation. In either case, additional views are required to determine the exact nature of the passage. Figure $4 \mathrm{c}$ demonstrates that as the resolution of the OG and OM approaches increases, the memory demands also substantially increase. By comparison, the GMM approach requires substantially less memory. When using the GMM approach, the resulting resampled point cloud is shown in Fig. 4d, where a hole in the data is visible. This approach is compared to OM with varying leaf sizes in Figs. $4 \mathrm{e}$ to $4 \mathrm{~g}$.

To obtain these results, a GMM was learned consisting of 100 components. Each component requires 10 floating point numbers which includes six floating point numbers to represent the symmetric covariance, three floating point numbers for the mean, and one floating point number to represent the mixing weight. Additional memory was used to represent the pose via six floating point numbers (three each for translation and rotation) where each floating point number is assumed to be four bytes. A 32-bit unsigned integer (four bytes) is also used to represent the support size of the GMM. In the OG case, one floating point number is used to store the logodds value and one unsigned integer (four bytes) is used to represent the index for each voxel in the change set. The total change set of $N$ voxels is transmitted along with meta-data to reconstruct the grid. The meta-data consists of three unsigned integers to represent the dimensions of the grid in width, height, and length as well as three floating point numbers to represent the origin for a total of 24 bytes. The 


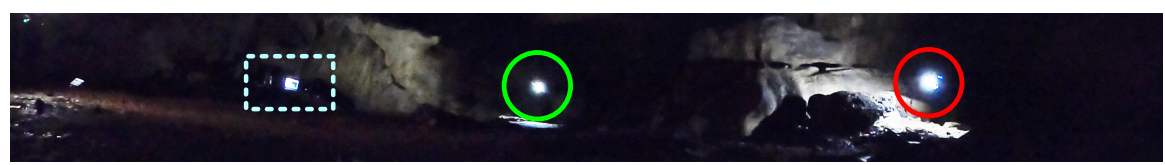

(a) Robots (circled) deployed in a cave. Communication router shown via dotted line.

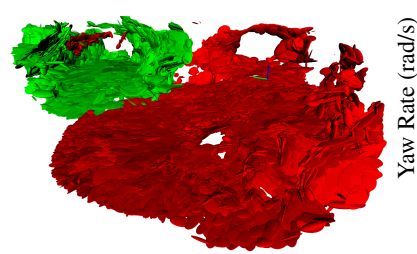

(b) Combined GMM map

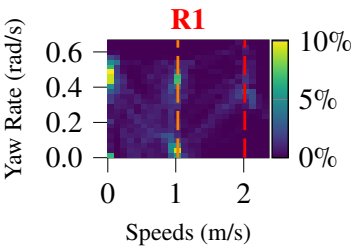

(c) Speed bounds shown by dashed lines.

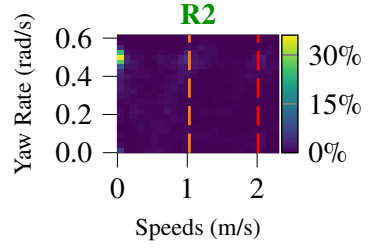

\begin{tabular}{ccccccc}
\hline Completion \%: & \multicolumn{2}{c}{$45 \%$} & \multicolumn{2}{c}{$65 \%$} & \multicolumn{2}{c}{$85 \%$} \\
\hline Case & $\begin{array}{c}\text { Map Size } \\
\text { (Mbit) }\end{array}$ & $\begin{array}{c}\text { Time } \\
\text { (hours) }\end{array}$ & $\begin{array}{c}\text { Map Size } \\
\text { (Mbit) }\end{array}$ & $\begin{array}{c}\text { Time } \\
\text { (hours) }\end{array}$ & $\begin{array}{c}\text { Map Size } \\
\text { (Mbit) }\end{array}$ & $\begin{array}{c}\text { Time } \\
\text { (hours) }\end{array}$ \\
\hline GMM & $\mathbf{0 . 7 \times \mathbf { 1 0 } ^ { \mathbf { 1 } }}$ & $\mathbf{8 . 0 \times \mathbf { 1 0 } ^ { - \mathbf { 3 } }}$ & $\mathbf{1 . 4 \times \mathbf { 1 0 } ^ { \mathbf { 1 } }}$ & $\mathbf{1 . 6 \times \mathbf { 1 0 } ^ { - 2 }}$ & $\mathbf{1 . 9} \times \mathbf{1 0}^{\mathbf{1}}$ & $\mathbf{2 . 2 \times 1 0 ^ { - 2 }}$ \\
OG $(0.1 \mathrm{~m})$ & $9.2 \times 10^{1}$ & $1.0 \times 10^{-1}$ & $1.57 \times 10^{2}$ & $1.7 \times 10^{-1}$ & $2.0 \times 10^{2}$ & $2.3 \times 10^{-1}$ \\
OG $(0.05 \mathrm{~m})$ & $5.4 \times 10^{2}$ & $6.0 \times 10^{-1}$ & $9.1 \times 10^{2}$ & $0.1 \times 10^{1}$ & $1.2 \times 10^{3}$ & $0.1 \times 10^{1}$ \\
OG $(0.025 \mathrm{~m})$ & $3.9 \times 10^{3}$ & $0.4 \times 10^{1}$ & $6.7 \times 10^{3}$ & $0.7 \times 10^{1}$ & $8.9 \times 10^{3}$ & $0.9 \times 10^{1}$ \\
OM $(0.1 \mathrm{~m})$ & $2.4 \times 10^{2}$ & $2.6 \times 10^{-1}$ & $3.9 \times 10^{2}$ & $4.4 \times 10^{-1}$ & $5.2 \times 10^{2}$ & $5.8 \times 10^{-1}$ \\
OM $(0.05 \mathrm{~m})$ & $1.6 \times 10^{3}$ & $0.2 \times 10^{1}$ & $2.6 \times 10^{3}$ & $0.3 \times 10^{1}$ & $3.4 \times 10^{3}$ & $0.4 \times 10^{1}$ \\
OM $(0.025 \mathrm{~m})$ & $9.8 \times 10^{3}$ & $1.1 \times 10^{1}$ & $1.6 \times 10^{4}$ & $1.8 \times 10^{1}$ & $2.1 \times 10^{4}$ & $2.4 \times 10^{1}$ \\
\hline
\end{tabular}

(d) Communication-Efficiency Comparison

Fig. 5: Rapid and communication efficient exploration of a cave with a team of two aerial robots. (a) illustrates the environment with the two robots ( $\mathbf{R} 1$ and $\mathbf{R} 2)$ and the WiFi router used for communication. (b) illustrates the final GMM maps generated on the base-station. (c) shows the percentage density plots for linear speeds and yaw rates as measured by the visual-inertial navigation system during flight. (d) highlights that the GMM approach requires significantly less memory to represent the combined map as compared to state-of-the-art approaches. In the context of transmitting this data using a channel with capacity $0.25 \mathrm{Mbit} / \mathrm{s}$, it would take significantly less time for the GMM approach as compared to the other approaches. A video of the flight can be accessed here: https://youtu.be/osko8EKKZUM.

total data required to represent the sensor observation with an $\mathrm{OG}$ is $8 N+24$ bytes. For OM, the full probabilistic model is serialized and stored to disk. The size of the file is reported in the table. The motivation for retaining the logodds values in the OG and OM representations is to enable information-theoretic planning. The advantage of the GMM approach is that the probability of occupancy can be reconstructed at an arbitrary voxel resolution [22,28], which significantly reduces the memory requirements as compared to the $\mathrm{OG}$ and $\mathrm{OM}$ approaches. The $\mathrm{OG}$ and $\mathrm{OM}$ approaches must retain the probability of occupancy to enable information-theoretic exploration $[24,29]$. 


\subsection{Hardware Experiments}

The second evaluation consists of hardware experiments for two aerial systems exploring the cave. The experiment demonstrates (1) each robot generates informative plans with linear speeds up to $2.37 \mathrm{~m} / \mathrm{s}$ and yaw rates up to $0.6 \mathrm{rad} / \mathrm{s}$ while maintaining safety and (2) the communication required to transmit the map from robots to a base station is substantially less as compared to the $\mathrm{OG}$ and $\mathrm{OM}$ approaches. For the purposes of this experiment, the robots are deployed in disjoint bounding boxes and the coordination between robots is not studied. What follows is a description of the experimental setup (including the implementation details) and results.

Each robot in the multi-robot system employs the navigation and control technique outlined in prior work [8]. The robots communicate with other computers on the network via WiFi and use the User Datagram Protocol (UDP) to transfer packets over the network. Before the start of each experiment, the SE(3) transform between the takeoff positions of the robots is measured manually using the navigation approach. The relative initial transform is used by the distributed mapping subsystem to align the GMM map fragments in the frames of other robots to the current robot's local frame.

The maximum speed ${ }^{4}$ of the robots in the xy-plane is $2.0 \mathrm{~m} / \mathrm{s}$, the maximum speed towards unknown space is $1.0 \mathrm{~m} / \mathrm{s}$, the maximum z-direction speed is $0.25 \mathrm{~m} / \mathrm{s}$, and the maximum yaw rate is constrained to $0.5 \mathrm{rad} / \mathrm{s}$. One of the metrics used to assess the planning performance is quantifying the maximum speed and yaw rate achieved by the robot while ensuring collision free operation. Both linear and yawing motions are exploratory actions for an aerial robot equipped with a limited field of view depth sensor $[8,10]$. The data transmitted from the robots to the base station is used to quantify the success of the mapping approach. The GMM results of Fig. 5 are generated in flight during an actual trial in the cave. To enable a fair comparison, the depth images collected from the GMM exploration trial in the cave are postprocessed using the $\mathrm{OG}$ and $\mathrm{OM}$ approaches. This ensures that variation in the other subsystems does not unduly affect the results. An analysis to quantify the memory required for each approach similar to Section 3.1 is presented. The OG and OM results are generated by updating the map using the depth information for the current image and publishing the change set. For the OM approach, the change set is serialized to file as the full probabilistic model to enable the base station and other robot to exactly recreate the map for information-theoretic exploration.

The two deployed robots are denoted by $\mathbf{R} \mathbf{1}$ and $\mathbf{R} \mathbf{2}$ in Fig. 5. The robots achieve high exploration rates by selecting actions that enable safe operation at linear speeds up to $2.37 \mathrm{~m} / \mathrm{s}$ and yaw rates up to $0.6 \mathrm{rad} / \mathrm{s}$, which are of the same order as state-ofthe-art fast exploration works ${ }^{5}[10,11,12]$. Moreover, note that since $\mathbf{R} 1$ operates

\footnotetext{
${ }^{4}$ The speed limits and the operational volumes were chosen based on the cave passage dimensions. The authors worked with cave management to select a test site that contained neither actively growing speleothems or bats. Possible effects of imperfect trajectory tracking and state estimation were also taken into account.

${ }_{5}^{5}$ The attained speeds exceed the limits slightly due to imperfect trajectory tracking and state estimation.
} 
in a relatively open space compared to $\mathbf{R} 2$, a larger percentage of high speed actions are selected (Fig. 5c). In contrast, the planner selects the yawing motion and slow linear actions towards frontiers more often for $\mathbf{R} 2$ to allow for safe operation in a constrained space (Fig. 5c). Both of these behaviors in the multi-robot system arise automatically due to the choice of the action representation for single-robot planning in [10]. These behaviors show that the same action representation can be used on every robot in the team without any change in parameters and still allow for intelligent speed adaptation for rapid and safe exploration.

The combined map from $\mathbf{R} \mathbf{1}$ and $\mathbf{R} 2$ requires significantly less time to transmit under the bandwidth constraint when measuring at various points during exploration (Fig. 5d). An implication of this in the context of the concept of operations is that at $100 \%$ exploration completion it will take about 104.40 seconds to transmit the GMM map, 12.30 hours to transmit the $0.025 \mathrm{~m}$ resolution OG map, and 1.25 days to transmit the $0.025 \mathrm{~m}$ resolution OM map to Earth. It is important to note why the OM approach requires more memory than the $\mathrm{OG}$ approach for this result while it required less memory than the OG approach in Fig. 4c. The change set must be encoded as an OctoMap before serializing to file. The approach presented by Hornung et al. [20] requires that the spatial relationships between nodes be implicitly stored in the encoding. This means that the serialized stream does not contain any $3 \mathrm{D}$ coordinates and additional data must be stored to preserve the structure of the octree. This is in contrast to the OG approach that stores a logodds value and index from which 3D coordinates can be recovered. Therefore, for small change sets, the OM approach has much higher overhead than the OG approach.

\subsection{Effects of Constrained Communication}

For this study the assumption on the robots operating in disjoint spaces is relaxed and a priority-based inter-robot collision checker is implemented for shared space operation. The simulation consists of a two-robot team that explores the cave environment. Two approaches are tested: GMM and OG. The OM approach is not compared for this experiment because to the best of our knowledge there is no existing open-source implementation of the Shannon mutual information used for planning by Zhang et al. [29]. Further, this enables us to retain the same planning subsystem for a fair comparison of the GMM and OG approaches. The communication rate is varied among $0.1 \mathrm{Mbit} / \mathrm{s}, 0.25 \mathrm{Mbit} / \mathrm{s}$, and unconstrained. Each configuration is tested in $40 \mathrm{ex}-$ periments with a $700 \mathrm{~s}$ duration. The duration of the exploration is chosen based on the top speed of the robots and the spatial dimensions of the environment. The exploration software is run on separate computers in a distributed fashion over a wired connection. The simulations are run on two desktop computers running Ubuntu 18.04 with Intel i7-6700K CPUs. One computer has 32 GB RAM and the other has $16 \mathrm{~GB}$ of RAM. For the wired connection, the data rate is limited via the network traffic control tool in Linux that uses the Token Bucket Filter (TBF) to maintain the specified rate value [30]. Figure 6 illustrates the results from the simulation study. 


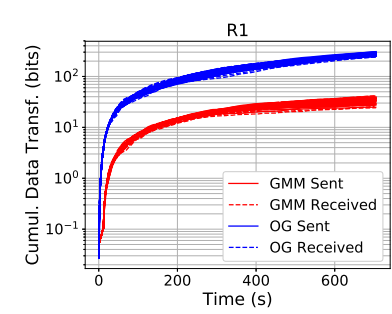

(a) No limit

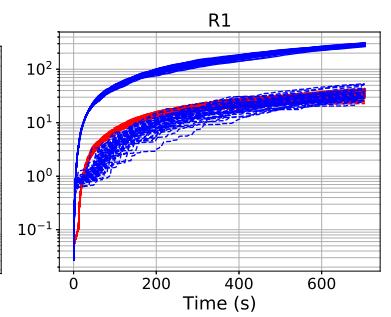

(b) $0.25 \mathrm{Mbit} / \mathrm{s}$

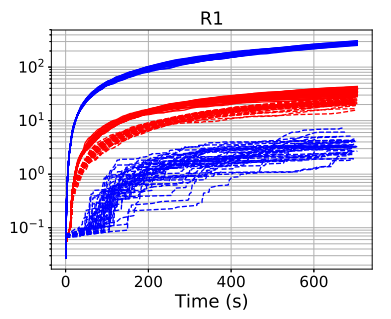

(c) $0.1 \mathrm{Mbit} / \mathrm{s}$

\begin{tabular}{cccccccccc}
\hline Completion \%: & \multicolumn{3}{c}{$45 \%$} & \multicolumn{3}{c}{$65 \%$} & \multicolumn{3}{c}{$85 \%$} \\
\hline Comm. Limit & $\begin{array}{c}\text { GMM } \\
(\mathrm{s})\end{array}$ & $\begin{array}{c}\text { OG } \\
(\mathrm{s})\end{array}$ & $\begin{array}{c}\Delta \\
(\%)\end{array}$ & $\begin{array}{c}\text { GMM } \\
(\mathrm{s})\end{array}$ & $\begin{array}{c}\text { OG } \\
(\mathrm{s})\end{array}$ & $\begin{array}{c}\Delta \\
(\%)\end{array}$ & $\begin{array}{c}\text { GMM } \\
(\mathrm{s})\end{array}$ & $\begin{array}{c}\text { OG } \\
(\mathrm{s})\end{array}$ & $\begin{array}{c}\Delta \\
(\%)\end{array}$ \\
\hline No limit & 80.19 & 81.51 & 1.62 & 130.73 & 131.98 & 0.95 & 225.8 & 237.51 & 4.93 \\
$0.25 \mathrm{Mbit} / \mathrm{s}$ & 79.91 & 92.38 & $\mathbf{1 3 . 5}$ & 129.1 & 160.15 & $\mathbf{1 9 . 3 9}$ & 214.86 & 282.11 & $\mathbf{2 3 . 8 4}$ \\
$0.1 \mathrm{Mbit} / \mathrm{s}$ & 86.51 & 93.43 & 7.41 & 142.95 & 165.88 & 13.82 & 247.83 & 270.15 & 8.27 \\
\hline
\end{tabular}

(d) Exploration completion times

Fig. 6: Variation of exploration performance with inter-robot communication limits. (a), (b), and (c) plot the cumulative map data sent and received for the GMM and OG approaches under different data rate constraints (the plots are shown for $\mathrm{R} 1$ only for brevity). The received data is impacted significantly for the $\mathrm{OG}$ approach at $0.25 \mathrm{Mbit} / \mathrm{s}$ while both approaches are affected at $0.1 \mathrm{Mbit} / \mathrm{s}$. Note that in all experiments the planning and coordination methodology is kept the same for a fair comparison. (d) compares the time to achieve a certain percentage of environment coverage. We observe that at the $0.25 \mathrm{Mbit} / \mathrm{s}$ constraint, the GMM approach improves the performance of the team by up to $23.84 \%$.

As the communication bandwidth is reduced from no limit in Fig. 6a to $0.25 \mathrm{Mbit} / \mathrm{s}$ the OG approach begins to drop packets and the exploration performance of the multi-robot approach decreases as compared to the GMM approach (see Fig. 6d). At this rate, the GMM approach achieves $85 \%$ environment coverage in less than $80 \%$ of the time that it takes the OG approach. However, as the communication rate decreases further to $0.1 \mathrm{Mbit} / \mathrm{s}$ the GMM approach also suffers though it is able to outperform the OG approach.

\section{Conclusion and Future Work}

This work leveraged the compactness of Gaussian mixture models for high-fidelity perceptual modeling to increase the rate of multi-robot exploration in reduced bandwidth scenarios such as autonomy in caves. The mapping approach enables retention of environment details while remaining amenable to low-bandwidth transmission. The advantage of this mapping strategy is that it enables a substantial increase in exploration rate of the multi-robot team as compared to state-of-the-art mapping 
techniques even as the communication bandwidth of the connection between robots decreases. Future work will improve perceptual detail in the environment and develop hierarchical strategies that adapt the fidelity of the model based on the sensor data. Multi-modal mapping (for example, thermal, RGB, etc.) may also be beneficial in these scenarios. Finally, coordination strategies can be developed to enable robots to share communication-efficient policies and improve the rate of exploration.

\section{References}

[1] Board SS, Council NR, et al. (2012) Vision and voyages for planetary science in the decade 2013-2022. National Academies Press

[2] Maimone M, Cheng Y, Matthies L (2007) Two years of visual odometry on the mars exploration rovers. Journal of Field Robotics 24(3):169-186

[3] Phillips-Lander C, et al. (2020) Macie: Mars astrobiological caves and internal habitability explorer (a new frontiers mission concept). LPI (2326):1142

[4] Stamenkovic V, et al. (2019) The next frontier for planetary and human exploration. Nature Astronomy 3(2):116-120

[5] Miranda D (2020) 2020 nasa technology taxonomy

[6] Whittaker R, et al. (2014) Exploration of planetary skylights and tunnels

[7] Cortés J, Egerstedt M (2017) Coordinated control of multi-robot systems: A survey. SICE Journal of Control, Measurement, and System Integration 10(6):495-503

[8] Tabib W, Goel K, Yao J, Boirum C, Michael N (2020) Autonomous cave surveying with an aerial robot. arXiv preprint arXiv:200313883

[9] Cieslewski T, Kaufmann E, Scaramuzza D (2017) Rapid exploration with multi-rotors: A frontier selection method for high speed flight. In: Proc. of the IEEE/RSJ Intl. Conf. on Intell. Robots and Syst., Vancouver, Canada

[10] Goel K, Corah M, Boirum C, Michael N (2019) Fast exploration using multirotors: Analysis, planning, and experimentation. In: Conf. on Field and Service Robot., Tokyo, Japan

[11] Dai A, Papatheodorou S, Funk N, Tzoumanikas D, Leutenegger S (2020) Fast frontier-based information-driven autonomous exploration with an mav. arXiv preprint arXiv:200204440

[12] Dharmadhikari M, Dang T, Solanka L, Loje J, Nguyen H, Khedekar N, Alexis $\mathrm{K}$ (2020) Motion primitives-based path planning for fast and agile exploration using aerial robots. In: 2020 IEEE International Conference on Robotics and Automation (ICRA), IEEE, pp 179-185

[13] Cesare K, Skeele R, Yoo SH, Zhang Y, Hollinger G (2015) Multi-uav exploration with limited communication and battery. In: 2015 IEEE international conference on robotics and automation (ICRA), IEEE, pp 2230-2235

[14] Corah M, O'Meadhra C, Goel K, Michael N (2019) Communication-efficient planning and mapping for multi-robot exploration in large environments. IEEE 
Robotics and Automation Letters 4(2):1715-1721, DOI 10.1109/LRA.2019. 2897368

[15] Matthies L (2017) Niac phase 1 final study report on titan aerial daughtercraft

[16] DARPA (2020) Darpa subterranean challenge. URL https://www. subtchallenge.com/

[17] Ebadi K, Chang Y, Palieri M, Stephens A, Hatteland A, Heiden E, Thakur A, Morrell B, Wood S, Carlone L, et al. (2020) Lamp: Large-scale autonomous mapping and positioning for exploration of perceptually-degraded subterranean environments. arXiv preprint arXiv:200301744

[18] Dang T, Tranzatto M, Khattak S, Mascarich F, Alexis K, Hutter M (2020) Graph-based subterranean exploration path planning using aerial and legged robots. Journal of Field Robotics

[19] Rouček T, Pecka M, Čížek P, Petříček T, Bayer J, Šalanskỳ V, Heřt D, Petrlík M, Báča T, Spurnỳ V, et al. (2019) Darpa subterranean challenge: Multirobotic exploration of underground environments. In: International Conference on Modelling and Simulation for Autonomous Systems, Springer, pp 274-290

[20] Hornung A, Wurm KM, Bennewitz M, Stachniss C, Burgard W (2013) Octomap: An efficient probabilistic 3d mapping framework based on octrees. Autonomous Robots 34(3):189-206

[21] Elfes A (1989) Using occupancy grids for mobile robot perception and navigation. IEEE Computer Society 22(6):46-57

[22] Tabib W, Goel K, Yao J, Dabhi M, Boirum C, Michael N (2019) Real-time information-theoretic exploration with gaussian mixture model maps. In: Proc. of Robot.: Sci. and Syst., FreiburgimBreisgau, Germany, DOI 10.15607/RSS. 2019.XV.061

[23] Chaslot G (2010) Monte-Carlo tree search. PhD thesis, Universiteit Maastricht

[24] Charrow B, Liu S, Kumar V, Michael N (2015) Information-theoretic mapping using Cauchy-Schwarz quadratic mutual information. In: Proc. of the IEEE Intl. Conf. on Robot. and Autom., Seattle, WA

[25] Cai C, Yang C, Zhu Q, Liang Y (2007) Collision avoidance in multi-robot systems. In: 2007 International Conference on Mechatronics and Automation, IEEE, pp 2795-2800

[26] Kisseleff S, Akyildiz IF, Gerstacker WH (2018) Survey on advances in magnetic induction-based wireless underground sensor networks. IEEE Internet of Things Journal 5(6):4843-4856

[27] NASA (2019) Mars science laboratory data rates/returns. URL https:// marsmobile.jpl.nasa.gov/msl/mission/communicationwithearth/data/

[28] O'Meadhra C, Tabib W, Michael N (2019) Variable resolution occupancy mapping using gaussian mixture models. IEEE Robot Autom Letters 4(2):20152022, DOI 10.1109/LRA.2018.2889348

[29] Zhang Z, Henderson T, Karaman S, Sze V (2020) Fsmi: Fast computation of shannon mutual information for information-theoretic mapping. The International Journal of Robotics Research 39(9):1155-1177 
[30] Hubert B, Graf T, Maxwell G, van Mook R, van Oosterhout M, Schroeder P, Spaans J, Larroy P (2002) Linux advanced routing \& traffic control. In: Ottawa Linux Symposium, sn, vol 213 